\title{
Comparing the Neuropsychological Test Performance of Operation Enduring Freedom/Operation Iraqi Freedom (OEF/OIF) Veterans with and without Blast Exposure, Mild Traumatic Brain Injury, and Posttraumatic Stress Symptoms
}

\author{
Daniel Storzbach, ${ }^{1,2,3}$ Maya Elin O’Neil, ${ }^{1,2,4}$ Saw-Myo Roost, ${ }^{1}$ Halina Kowalski, ${ }^{1}$ Grant L. Iverson, ${ }^{5,6,7}$ \\ Laurence M. Binder, ${ }^{2,3,8}$ Jesse R. Fann, ${ }^{9,10}$ AND Marilyn Huckans ${ }^{1,2}$ \\ ${ }^{1}$ Portland VA Medical Center, Portland, Oregon \\ ${ }^{2}$ Oregon Health \& Science University Department of Psychiatry, Portland, Oregon \\ ${ }^{3}$ Oregon Health \& Science University Department of Neurology, Portland, Oregon \\ ${ }^{4}$ Oregon Health \& Science University Department of Medical Informatics \& Clinical Epidemiology, Portland, Oregon \\ ${ }^{5}$ Department of Physical Medicine and Rehabilitation, Harvard Medical School, Boston, Massachusetts \\ ${ }^{6}$ Red Sox Foundation and Massachusetts General Hospital Home Base Program, Boston, Massachusetts \\ ${ }^{7}$ Defense and Veterans Brain Injury Center, Bethesda, Maryland \\ ${ }^{8}$ Independent practice, Beaverton, Oregon \\ ${ }^{9}$ Department of Psychiatry and Behavioral Sciences, University of Washington, Seattle, Washington \\ ${ }^{10}$ Department of Rehabilitation Medicine, University of Washington, Seattle, Washington
}

(Received August 19, 2014; Final Revision April 9, 2015; Accepted April 13, 2015; First Published OnLine June 1, 2015)

\begin{abstract}
To compare neuropsychological test performance of Veterans with and without mild traumatic brain injury (MTBI), blast exposure, and posttraumatic stress disorder (PTSD) symptoms. We compared the neuropsychological test performance of 49 Operation Enduring Freedom/Operation Iraqi Freedom (OEF/OIF) Veterans diagnosed with MTBI resulting from combat blast-exposure to that of 20 blast-exposed OEF/OIF Veterans without history of MTBI, 23 OEF/OIF Veterans with no blast exposure or MTBI history, and 40 matched civilian controls. Comparison of neuropsychological test performance across all four participant groups showed a complex pattern of mixed significant and mostly nonsignificant results, with omnibus tests significant for measures of attention, spatial abilities, and executive function. The most consistent pattern was the absence of significant differences between blast-exposed Veterans with MTBI history and blast-exposed Veterans without MTBI history. When blast-exposed Veteran groups with and without MTBI history were aggregated and compared to non-blast-exposed Veterans, there were significant differences for some measures of learning and memory, spatial abilities, and executive function. However, covariation for severity of PTSD symptoms eliminated all significant omnibus neuropsychological differences between Veteran groups. Our results suggest that, although some mild neurocognitive effects were associated with blast exposure, these neurocognitive effects might be better explained by PTSD symptom severity rather than blast exposure or MTBI history alone. (JINS, 2015, 21, 353-363)
\end{abstract}

Keywords: Brain injuries, Blast injuries, Explosions, Military personnel, Combat disorders, Stress disorders, Post-traumatic, Cognitive symptoms

\section{INTRODUCTION}

Since the onset of the recent conflicts in Afghanistan and Iraq, the effects of combat-related blast exposure and traumatic brain injury (TBI) have been a source of increasing

Correspondence and reprint requests to: Maya Elin O'Neil, Portland VA Medical Center (R\&D 66), 3710 SW US Veterans Hospital Road, Portland, OR 97239-2964. E-mail: maya.oneil@va.gov concern to the Department of Defense, the Department of Veterans Affairs, and the public at-large. However, the neuropsychological sequelae specific to combat blast exposure remain unclear. Furthermore, because combat-deployed personnel are exposed to multiple cognitive risk factors such as environmental pollutants and physical and psychological stress, blast exposure and TBI are not the only potential sources of combat-related cognitive effects (Vasterling \& Proctor, 2011). In particular, posttraumatic stress disorder 
(PTSD) is very common in Operation Enduring Freedom/ Operation Iraqi Freedom (OEF/OIF)-deployed personnel, and is also associated with cognitive difficulties (Verfaellie \& Vasterling, 2009).

Several studies on the neuropsychological effects of combat-related blast exposure in OEF/OIF combat personnel have focused on whether cognitive effects differ for Veterans with history of blast-related compared to non-blast-related mild TBI (MTBI). When Belanger and colleagues compared patterns of performance on neuropsychological tests in subjects who sustained TBIs as a result of blast exposure with those who sustained TBIs from non-blast exposure (e.g., blunt force trauma), they found few differences (Belanger, Kretzmer, Yoash-Gantz, Pickett, \& Tupler, 2009). Similarly, Luethcke et al. compared blast versus non-blast MTBI (including injuries from secondary, tertiary, and quarternary blast exposure in the non-blast group) based on neuropsychological evaluations conducted within $72 \mathrm{hr}$ of injury and found clinically significant impairment in cognitive reaction time for both groups, but no cognitive differences between blast versus non-blast injury (Luethcke, Bryan, Morrow, \& Isler, 2010). Additionally, Brenner et al. (2010) compared neuropsychological test performance of soldiers with a history of blast-related MTBI and continuing MTBI symptoms to that of soldiers without MTBI symptoms an average of 42 weeks after injury, and found no statistically significant impact of the presence of MTBI symptoms on test performance. Finally, MacDonald et al. (2014) report similar neuropsychological test performance when comparing means for groups of blast exposed and non-blast exposed evacuated U.S. military personnel with and without impact-related TBI history. They describe greater proportions of participants with clinically significant impairment than would be expected due to chance in those with TBI history regardless of blast exposure. Overall, although limited in their scope and methods, these few studies have not supported neuropsychological effects unique to blast-related MTBI in OEF/OIF personnel, but because many Veterans are exposed to blast without necessarily experiencing a TBI, the unique impact of combat blast exposure is unknown. No studies have specifically compared cognition in Veterans with blast exposure but no TBI to Veterans with blast-related MTBI, Veterans with no blast exposure, and civilian controls. Similarly, no studies have compared Veterans' cognitive test performance based on exposure to blast regardless of TBI history.

Combat blast exposure sometimes is a psychological as well as a physical trauma. With that in mind, researchers have also investigated the effects of blast-related mental health comorbidities, particularly PTSD, on neuropsychological complaints and performance in Veterans. Nelson and colleagues (2012) found that cognitive impairment was associated with Axis I diagnoses, particularly PTSD and depression. Lange et al. (2012) similarly found that neuropsychological effects of MTBI were no longer significant after controlling for psychological distress. Postconcussion symptoms, including subjective cognitive complaints, have also been found to be strongly associated with PTSD and other sources of psychological distress (Belanger, Kretzmer, Vanderploeg, \& French, 2009; Hoge et al., 2008; Lippa, Pastorek, Benge, \& Thorton, 2010). Most recently, Verfaellie, Lafleche, Spiro, and Bousquet (2014) reported that, among a cohort of Veterans exposed to blast, MTBI and loss of consciousness (LOC) did not affect cognitive performance; however, they found that cognitive performance was significantly related to both PTSD and depression in this cohort (Verfaellie et al., 2014). Although recent research suggests that PTSD symptoms may mediate associations between MTBI and cognitive test performance, no studies have examined the role of PTSD symptoms in the relationship between blast exposure and cognitive test performance.

The primary aim of this study (Aim 1) was to compare the neuropsychological test performance of blast-exposed OEF/ OIF Veterans who met current Department of Defense (DoD)/Department of Veterans Affairs (DVA) MTBI criteria to those without blast exposure or MTBI. We compared the neuropsychological test performance of blast-exposed OEF/ OIF Veterans with MTBI to that of blast-exposed OEF/OIF Veterans without MTBI, OEF/OIF Veterans with no blast exposure or TBI history, and civilian controls subjects. We hypothesized that the civilian controls and non-blastexposed Veterans would perform better than both blastexposed Veteran groups, and that those with MTBI history would perform worse than those without. Because the primary results suggest that there is an absence of significant differences between blast-exposed Veterans with and without MTBI history, a post hoc secondary aim (Aim 2) was to compare the neuropsychological test performance of deployed Veterans with and without blast exposure regardless of MTBI history, as we hypothesized that the latter group would perform better on neuropsychological tests. The final aim (Aim 3) was to examine the role of PTSD symptoms in the relationship between blast exposure and neuropsychological test performance. We hypothesized that PTSD symptoms would explain some of the variability in test performance between these two groups of Veterans.

\section{METHOD}

\section{Participants}

Study participants were OEF/OIF Veterans enrolled at the Portland Veterans Administration Medical Center (PVAMC) between September 2008 and April 2011, who were recruited in accordance with PVAMC Institutional Review Board (IRB) approval. Recruitment methods included posters, flyers, clinic referrals, word of mouth, and recruitment letters sent to Veterans who had undergone standardized Department of Veterans Affairs (DVA) TBI screening at the PVAMC, but method of recruitment was not included as part of data collection and, therefore, we do not have data on how many Veterans responded to which type of recruitment method. Participants were included in the study if they were: (1) OEF/OIF Veterans who were able to provide informed consent; and (2) native English speakers (to preclude any effect of language 
differences on verbal tests). Participants were excluded from the study if they: (1) met Diagnostic and Statistical Manual of Mental Disorders, $4^{\text {th }}$ Edition (DSM-IV; American Psychiatric Association, 2000) criteria for current substance abuse or dependence and had been substance abstinent for less than 30 days, reported use of illicit drugs within the past $24 \mathrm{hr}$, reported any history of methamphetamine abuse, or reported use of alcohol or alcohol intoxication within the past $24 \mathrm{hr}$; (2) reported a history of TBI with LOC or posttraumatic amnesia (PTA) before or after OIF or OEF deployment; or, TBI during OIF or OEF deployment from injury other than explosion; or PTA greater than $24 \mathrm{hr}$ or LOC greater than $30 \mathrm{~min}$; (3) reported a history of schizophrenia, schizoaffective disorder, or other psychotic disorder; (4) reported a history of a medical condition associated with persisting cognitive problems or serious central nervous system dysfunction (e.g., brain tumor, multiple sclerosis, dementia, seizure disorder, human immunodeficiency virus infection, hepatitis C infection); (5) reported a history of auditory or visual impairments that would prevent completion of cognitive tests; and (6) obtained scores indicative of possible inadequate effort or motivation, defined as scores of less than 48 on the Test of Memory Malingering Trial 2 (TOMM). We used a TOMM cutoff score more stringent than the score originally recommended by the test author for use across all possible neurodiagnostic groups (Tombaugh, 1996) because individuals with MTBI providing valid responses do not score below the higher cutoff score (Greve, Ord, Curtis, Bianchini, \& Brennan, 2008; Tombaugh, 1996).

Based on the above criteria, one participant was excluded due to being a non-native speaker of English, two participants were excluded due to substance use, one was excluded due to visual impairment, and 10 participants were excluded due to obtaining TOMM scores falling below the cutoff points (of the 10, six had Trial 2 scores between 45 and 47, and 4 had scores below 45). Additionally, four participants did not complete any of the cognitive testing after completing the clinical interview, and their incomplete data were excluded from the analyses.

\section{Veteran Grouping}

Veteran participants were divided into three groups related to blast exposure during deployment to Iraq and/or Afghanistan. The three groups were: (1) the "Non-Blast-Exposed No TBI" (NBNTBI) group, consisting of OEF/OIF-deployed Veterans who reported that they had not experienced blast exposure nor TBI during their deployment; (2) the "Blast-Exposed No TBI" (BNTBI) group, consisting of OEF/OIF-deployed Veterans who self-reported exposure to one or more explosions during their deployment which were not associated with acute MTBI symptoms and did not meet DoD/DVA criteria for diagnosis of MTBI; and (3) the "Blast-Exposed MTBI" (BMTBI) group, consisting of OEF/OIF-deployed Veterans who self-reported a history of $0.5 \mathrm{hr}$ or less of LOC and/or $24 \mathrm{hr}$ or less of PTA or alteration of consciousness/ mental state after blast consistent with DoD/DVA criteria for diagnosis of MTBI. Blast exposure was determined by a 2-step procedure: (1) medical record review of post deployment TBI screening; (2) a semi-structured interview of the Veteran querying exposure details including proximity to the blast and whether or not the Veteran was hit by debris or suffered other direct impact to the head. Even if medical record review was negative for blast exposure, all Veteran participants were asked about blast exposure during the clinical interview; no distance from blast criteria were applied, and all Veterans who reported exposure to blast and associated effects (e.g., feeling "blast waves" or getting knocked over by an explosion) were classified as blast-exposed.

\section{Civilian Controls from the NAB Normative Sample}

A civilian comparison group (CIV) was selected from the Neuropsychological Assessment Battery (NAB) normative sample. The selection of control cases was matched as closely as possible with the other groups based on age, gender, level of education, and an estimate of intellectual functioning as determined by the Reynolds Intellectual Screening Test (RIST) Index score.

\section{Procedures and Instruments}

Veteran participants completed evaluation sessions that lasted approximately 4 to $6 \mathrm{hr}$ and were compensated $\$ 50$. All subjects were carefully screened via clinical judgment to ensure their ability to comprehend study procedures, risks, and benefits and provided informed consent to participate in the study in accordance with PVAMC IRB-approval. Each session included the procedures listed below.

\section{Medical Record Review}

Each Veteran's medical record was reviewed to document psychiatric and medical history, current treatment, and medications. The medical record review was unstructured and designed to gather any information relevant to TBI history. Information obtained during medical records review was not communicated to study personnel administering neuropsychological tests.

\section{Clinical Interview}

Clinical interviews were administered by doctoral level clinical psychologists experienced in the evaluation of TBI. Veteran participants were administered a structured diagnostic interview, the Mini International Neuropsychiatric Interview (MINI; Sheehan, Shytle, Milo, Janavs, \& Lecrubier, 2006). Mental health diagnoses based on the MINI and diagnoses clearly documented in medical charts were used as the basis for mental health diagnostic exclusion criteria. A review of medical and medication history, blast exposure, TBI history and symptoms, and other relevant data including age, gender, education, and socio-economic status was collected for each participant as part of a structured interview designed for this study. TBI history interview items were designed to adhere to VA/DOD TBI criteria. Information obtained during clinical interviews was not communicated to study personnel 
administering neuropsychological tests. TBI history was established by both medical record review and clinical interview. It was common for the information in the medical record to be incomplete or different from the clinical interview, in which case we relied upon the information gathered during the interview to establish TBI history.

\section{Neurocognitive Assessment Battery}

Study personnel, blinded to Veterans' history and group membership, administered the neuropsychological tests to Veteran participants according to published, standardized procedures. The following tests were included in the battery: (1) The Wechsler Test of Adult Reading (WTAR; Wechsler, 2001) assesses basic oral reading skills and was used to estimate pre-injury intellectual functioning. (2) The Reynolds Intellectual Screening Test (RIST; Reynolds \& Kamphaus, 2003) assesses fund of knowledge and non-verbal reasoning abilities. It was used to estimate pre-injury intellectual functioning. (3) An abbreviated version of the Neuropsychological Assessment Battery Memory Module (NAB - Memory Module; Stern \& White, 2003), which included list learning, story learning, and daily living memory. All tests in the module assess immediate and delayed recall. (4) An abbreviated version of the NAB Attention Module, which includes Digits Forward, Digits Backward, Dots, Numbers \& Letters A, B, C, and D, and Driving, assessing visual attention, auditory attention, and complex divided attention. (5) An abbreviated version of the NAB Executive Functions Module, which includes Mazes, Categories, and Word Generation. These tests measure planning, concept formation, and word generation. (6) Design Construction, from the NAB Spatial Module, measures visual-spatial problemsolving abilities. (7) The Test of Memory Malingering (TOMM; Tombaugh, 1996) assesses for suboptimal effort. For the NAB Memory, Attention, and Executive Function modules, in addition to specific subscale analyses, we also analyzed omnibus measures. We did not administer any NAB modules in their entirety; therefore, index scores were not available.

The test battery also included psychological assessment measures and symptom checklists, analysis of which is planned for publication in subsequent papers. This study reports results from the PTSD Checklist-Military Version (PCL-M; Weathers \& Ford, 1996), a 17-item self-report questionnaire based on DSM-IV diagnosis of PTSD that assesses symptom severity, usually requiring 5-7 min to administer. Coefficient alphas are .89-.97 for DSM criteria in Veteran samples, with good convergent and discriminant validity (Weather, Litz, Herman, Huska, \& Keane, 1993).

\section{Statistical Analyses}

To examine potential differences between groups on key demographic variables, we conducted analysis of variance (ANOVA) tests to compare mean age, education, RIST, and WTAR scores for the four groups of participants. We conducted the following analyses without including gender as a covariate because the very small number of women in our sample limited statistical power for these subgroup analyses; however, we conducted sensitivity analyses by only analyzing data on male participants and found no changes in the results in terms of both statistical significance or direction of effect. Because standard interpretation of neuropsychological test results includes adjustment for age and education, we analyzed data adjusting for these variables, although we present both raw and standard scores for comparison purposes. We also conducted sensitivity analyses examining how results differed when adjusting for RIST (all groups) and WTAR scores (all groups except for the civilian controls), and found no differences in results in terms of statistical significance or direction of effect.

Multivariate analysis of variance (MANOVA) and multivariate analysis of covariance (MANCOVA) tests were used to examine group differences on neurocognitive assessments. Separate MANCOVAs were conducted for each group of neurocognitive assessments composed of multiple tests assessing the same neurocognitive construct (e.g., Attention, Executive Functioning). The "Spatial Abilities" domain was assessed by only one neurocognitive test and was, therefore, analyzed using ANOVA and ANCOVA. The "Learning and Memory" domain was assessed by five different individual tests (e.g., List Learning, Story Memory, etc.) which were each composed of multiple scores (e.g., Immediate Recall, Short Delay Recall, etc.). For this construct, multivariate analyses were completed separately for the sub-domains of "Immediate Recall" and "Delayed Recall." We analyzed raw scores to maximize variance; however, we present results for both unadjusted raw and standardized scores to facilitate comparisons across samples and to enable estimation of clinical significance. Sensitivity analyses indicated no significant differences for analyses conducted with raw versus standardized scores. We covaried for age and education in all analyses to adjust for these known confounders, although sensitivity analyses indicated that results did not differ in terms of statistical significance or direction of effect when these covariates were not included. The first set of analyses compared results across all four groups of Veterans and civilians. The second set of analyses comparing blast exposed to non-blast exposed Veterans excluded the civilian sample, although sensitivity analyses yielded similar results when we included our civilian sample with the non-blast exposed group. Similarly, we included only Veteran samples in the last set of analyses comparing blast exposed and non-blast exposed Veterans after adjusting for PTSD symptoms because we did not have access to a measure of PTSD for the civilian sample.

\section{RESULTS}

There were no statistically significant differences among groups for age in years, $F(3,131)=1.36 ; p=.26$; years of education, $F(3,131)=2.28 ; p=.08$; or RIST scores, $F(3,131)=1.09 ; p=.36$. Table 1 summarizes these results and presents mean scores for the groups. Our sample was primarily male Veterans who were, on average, 33 years of age, with 13.5 years of education. We examined proportional gender differences across groups, which were statistically 
significant $\left(\chi^{2}=14.14 ; p<.01\right)$, with $30.4 \%, 5.0 \%, 2.0 \%$, and $12.5 \%$ women comprising the NBNTBI, BNTBI, BMTBI, and CIV groups, respectively. The BNTBI group experienced, on average, 8.53 blast exposures $(S D=9.90$, minimum $=1$, maximum $=30$ ) and the BMTBI group experienced an average of 8.33 blast exposures $(S D=15.97$, minimum $=1$, maximum $=95$ ). The average time since most serious TBI was 52.64 months $(S D=31.72)$ for the BNTBI group and 44.40 months $(S D=19.33)$ for the BMTBI group. In the BMTBI group, 22 Veterans reported LOC, 17 reported no LOC, and 10 reported possible LOC; 43 reported disorientation or confusion, 2 reported no disorientation or confusion, and 4 reported possible disorientation or confusion; and 21 reported PTA, 11 reported no PTA, and 17 reported possible PTA. In the BNTBI group, 19 Veterans reported no LOC and 1 reported possible LOC; 18 reported no disorientation or confusion and 2 reported possible disorientation or confusion; and 16 reported no PTA and 4 reported possible PTA. There were statistically significant differences between groups based on PCL score (see Table 1); the NBNTBI group had significantly lower PCL scores than the BNTBI and BMTBI groups ( $p=.01$ and $<.01$, respectively), although there were no significant differences in PCL scores for the BNTBI and BMTBI groups $(p=.07)$. Finally, we evaluated the assumption of homogeneity of variances for all analyses, and only the NAB Name, Address, Phone subtest violated this assumption; therefore, these results should be interpreted with caution.

\section{Aim 1}

We first compared mean scores on neurocognitive tests for all four groups of participants, presented in Table 2. These MANCOVA and ANCOVA results indicate non-significant

Table 1. Group comparisons

\begin{tabular}{|c|c|c|c|c|c|c|c|}
\hline Group & $N$ & Mean & $S D$ & Min & Max & $\mathrm{F}$ & $p$ \\
\hline & \multicolumn{7}{|c|}{ Age in years } \\
\hline No Blast Exposure, no TBI & 23 & 36.04 & 11.50 & 23 & 55 & & \\
\hline Blast Exposed, no TBI & 20 & 34.45 & 10.37 & 24 & 60 & & \\
\hline Blast Exposed with MTBI & 49 & 31.53 & 7.69 & 23 & 49 & & \\
\hline Combined Veteran sample & 92 & 33.29 & 9.46 & 23 & 60 & & \\
\hline Civilians & 40 & 33.42 & 9.25 & 18 & 50 & & \\
\hline \multirow[t]{2}{*}{ Total } & 132 & 33.33 & 9.36 & 18 & 60 & 1.36 & .26 \\
\hline & \multicolumn{7}{|c|}{ Years of education } \\
\hline No Blast Exposure, no TBI & 23 & 14.09 & 2.09 & 9 & 18 & & \\
\hline Blast Exposed, no TBI & 20 & 13.40 & 1.82 & 11 & 18 & & \\
\hline Blast Exposed, with MTBI & 49 & 13.04 & 1.70 & 9 & 19 & & \\
\hline Combined Veteran sample & 92 & 13.38 & 1.86 & 9 & 19 & & \\
\hline Civilians & 40 & 13.80 & 1.73 & 11 & 18 & & \\
\hline \multirow[t]{2}{*}{ Total } & 132 & 13.51 & 1.82 & 9 & 19 & 2.28 & .08 \\
\hline & \multicolumn{7}{|c|}{ Reynolds Intellectual Screening Test } \\
\hline No Blast Exposure, no TBI & 23 & 110.61 & 9.51 & 93 & 127 & & \\
\hline Blast Exposed, no TBI & 20 & 109.15 & 8.85 & 91 & 126 & & \\
\hline Blast Exposed with MTBI & 49 & 107.65 & 8.03 & 91 & 122 & & \\
\hline Combined Veteran sample & 92 & 108.72 & 8.59 & 91 & 127 & & \\
\hline Civilians & 40 & 106.83 & 8.66 & 91 & 122 & & \\
\hline \multirow[t]{2}{*}{ Total } & 132 & 108.14 & 8.62 & 91 & 127 & 1.09 & .36 \\
\hline & \multicolumn{7}{|c|}{ Wechsler Test of Adult Reading } \\
\hline No Blast Exposure, no TBI & 22 & 37.27 & 5.18 & 28 & 45 & & \\
\hline Blast Exposed, no TBI & 20 & 35.80 & 5.76 & 23 & 46 & & \\
\hline Blast Exposed with MTBI & 49 & 33.65 & 6.90 & 12 & 46 & & \\
\hline \multirow[t]{2}{*}{ Total (Combined Veteran sample) } & 91 & 35.00 & 6.41 & 12 & 46 & 2.73 & .071 \\
\hline & \multicolumn{7}{|c|}{ PTSD Checklist - Military Version } \\
\hline No Blast Exposure, no TBI & 23 & 30.61 & 13.99 & 17 & 69 & & \\
\hline Blast Exposed, no TBI & 20 & 44.95 & 18.17 & 17 & 85 & & \\
\hline Blast Exposed with MTBI & 47 & 54.72 & 15.53 & 18 & 82 & & \\
\hline Total (Combined Veteran sample) & 90 & 46.39 & 18.57 & 17 & 85 & 18.14 & $<.001$ \\
\hline
\end{tabular}

Note. MTBI $=$ mild traumatic brain injury, $\mathrm{PTSD}=$ posttraumatic stress disorder. 
Table 2. Neuropsychological Assessment Battery (NAB) mean raw score differences for veterans with and without blast exposure and MTBI, and civilian controls

\begin{tabular}{|c|c|c|c|c|c|c|c|c|c|c|c|}
\hline & $\mathrm{F}$ & $p$-Value & Partial $\eta^{2}$ & Civilian controls & $\begin{array}{c}\text { No TBI, No } \\
\text { blast exposure }\end{array}$ & $\begin{array}{c}\text { Blast exposed } \\
\text { No TBI }\end{array}$ & $\begin{array}{c}\text { Blast exposed } \\
\text { With MTBI }\end{array}$ & Civilian controls & $\begin{array}{l}\text { No TBI, No } \\
\text { blast exposure }\end{array}$ & $\begin{array}{c}\text { Blast exposed } \\
\text { No TBI }\end{array}$ & $\begin{array}{c}\text { Blast exposed } \\
\text { With MTBI }\end{array}$ \\
\hline & \multicolumn{3}{|c|}{ Learning \& Memory } & \multicolumn{4}{|c|}{ Unadjusted raw score mean (SD) } & \multicolumn{4}{|c|}{ T-Score mean $(S D)$} \\
\hline Immediate Recall & 1.41 & 0.16 & 0.04 & & & & & & & & \\
\hline List Learning & 2.36 & 0.08 & 0.05 & $26.13(4.35)$ & $25.78(3.38)$ & $23.70(3.99)$ & $23.96(4.40)$ & $52.20(11.02)$ & $50.91(7.17)$ & $47.35(9.60)$ & $47.41(9.29)$ \\
\hline Story Memory & 2.05 & 0.11 & 0.05 & $62.10(11.49)$ & $63.61(12.11)$ & $57.10(13.40)$ & $58.20(10.51)$ & $49.65(9.87)$ & $51.43(10.72)$ & $45.56(10.31)$ & $46.12(9.82)$ \\
\hline Daily Living Memory & 1.69 & 0.17 & 0.04 & $43.78(5.93)$ & $46.65(4.68)$ & $44.45(3.41)$ & $43.88(5.33)$ & $50.45(11.77)$ & $57.91(9.97)$ & $51.30(7.23)$ & $50.78(11.28)$ \\
\hline Medication Instructions & 1.16 & 0.33 & 0.03 & $23.78(3.32)$ & $25.17(2.73)$ & $24.45(2.31)$ & $24.14(2.94)$ & $48.36(11.26)$ & $53.10(9.23)$ & $50.64(7.81)$ & $49.60(9.97)$ \\
\hline Name, Address, Phone & 1.43 & 0.24 & 0.03 & $20.00(3.17)$ & $21.48(2.63)$ & $20.00(2.27)$ & $19.73(3.74)$ & $49.50(9.82)$ & $54.09(8.14)$ & $49.50(7.04)$ & $48.68(11.59)$ \\
\hline Delayed Recall & 1.56 & 0.08 & 0.06 & & & & & & & & \\
\hline List Learning, Short Delay & 3.87 & $0.01 *$ & 0.08 & $9.15(2.16)^{* \dagger}$ & $8.61(1.83)$ & $7.60(1.76)^{*}$ & $7.88(2.10) \dagger$ & $54.10(10.44)^{* \dagger}$ & $51.78(9.37)$ & $47.05(9.81)^{*}$ & $48.31(11.12) \dagger$ \\
\hline List Learning, Long Delay & 3.44 & $0.02 *$ & 0.08 & $8.85(2.35)^{*}$ & $8.57(2.39)$ & $7.25(2.27)$ & $7.39(2.58)^{*}$ & $53.53(11.13)^{*}$ & $52.26(11.42)$ & $45.85(11.09)$ & $46.51(13.56)^{*}$ \\
\hline Story Memory & 2.58 & 0.06 & 0.06 & $32.58(7.18)$ & $34.22(4.78)$ & $31.30(6.29)$ & $30.27(5.68)$ & $49.83(10.13)$ & $51.56(8.19)$ & $47.30(8.89)$ & $45.14(9.13)$ \\
\hline Daily Living Memory & 2.24 & 0.09 & 0.05 & $15.70(1.65)$ & $15.91(2.00)$ & $15.00(2.03)$ & $14.55(2.59)$ & $52.58(7.57)$ & $54.09(6.91)$ & $48.65(11.63)$ & $46.53(13.19)$ \\
\hline Medication Instructions & 0.30 & 0.83 & 0.01 & $8.45(0.90)$ & $8.43(1.12)$ & $8.40(0.82)$ & $8.20(1.24)$ & $50.94(8.51)$ & $50.80(10.55)$ & $50.47(7.72)$ & $48.63(11.68)$ \\
\hline \multirow[t]{2}{*}{ Name, Address, Phone } & 3.75 & $0.01 *$ & 0.08 & $7.25(1.06)$ & $7.48(1.08)^{*}$ & $6.60(1.31)$ & $6.35(1.77)^{*}$ & $52.66(7.19)$ & $54.21(7.37)^{*}$ & $48.23(8.95)$ & $46.51(12.09)^{*}$ \\
\hline & \multicolumn{3}{|c|}{ Attention } & & & & & & & & \\
\hline Digit Span & 3.84 & $0.01 *$ & 0.08 & & & & & & & & \\
\hline Digits forward & 6.53 & $<0.01 *$ & 0.14 & $10.28(2.01)^{*}$ & $9.30(2.40)$ & $8.80(1.94)$ & $8.06(2.55)^{*}$ & $54.10(9.28)^{*}$ & $50.17(11.12)$ & $48.00(8.23)$ & $44.24(11.40)^{*}$ \\
\hline Digits backward & 1.46 & 0.23 & 0.03 & $6.15(3.11)$ & $6.30(2.79)$ & $4.90(2.27)$ & $5.16(2.66)$ & $53.38(11.44)$ & $54.35(10.43)$ & $49.30(10.20)$ & $50.02(10.59)$ \\
\hline Numbers and Letters & 2.29 & $0.04^{*}$ & 0.05 & & & & & & & & \\
\hline C efficiency & 3.46 & $0.02 *$ & 0.08 & $71.90(18.46)^{*}$ & $65.14(23.10)$ & $63.05(17.46)$ & $56.27(21.82)^{*}$ & $51.70(8.26)^{*}$ & $49.43(11.15)$ & $47.65(8.67)$ & $44.84(8.91)^{*}$ \\
\hline \multirow[t]{2}{*}{ D efficiency } & 2.16 & 0.10 & 0.05 & $54.23(14.44)$ & $49.91(10.26)$ & $48.05(9.83)$ & $48.57(9.14)$ & $50.53(9.33)$ & $48.70(9.19)$ & $46.30(10.35)$ & $45.51(9.29)$ \\
\hline & \multicolumn{3}{|c|}{ Spatial } & & & & & & & & \\
\hline \multirow[t]{2}{*}{ Design construction } & 5.64 & $<0.01^{*}$ & 0.12 & $53.20(6.36)^{*}$ & $58.35(4.85)^{* \dagger}$ & $54.80(7.60)$ & $53.51(6.38) \dagger$ & $48.18(8.46)^{*}$ & $56.96(8.75)^{* \dagger}$ & $51.60(9.93)$ & $53.37(9.16) \dagger$ \\
\hline & \multicolumn{3}{|c|}{ Executive Functioning } & & & & & & & & \\
\hline Executive Functioning & 1.93 & $0.05^{*}$ & 0.04 & & & & & & & & \\
\hline Mazes & 0.76 & 0.52 & 0.02 & $18.63(5.85)$ & $19.87(4.48)$ & $19.40(6.45)$ & $20.00(5.59)$ & $49.05(9.48)$ & $52.09(7.15)$ & $50.75(8.73)$ & $49.78(9.05)$ \\
\hline Categories & 3.41 & $0.02 *$ & 0.08 & $22.33(7.46)^{*}$ & $27.61(7.78)^{*}$ & $23.55(8.45)$ & $24.04(6.62)$ & $49.70(8.03)^{*}$ & $55.65(8.42)^{*}$ & $52.05(6.86)$ & $51.27(6.93)$ \\
\hline Word generation & 1.34 & 0.27 & 0.03 & $12.20(4.49)$ & $11.30(3.02)$ & $10.15(3.59)$ & $10.61(3.87)$ & $51.28(10.68)$ & $49.70(6.09)$ & $47.40(7.36)$ & $48.20(8.60)$ \\
\hline
\end{tabular}

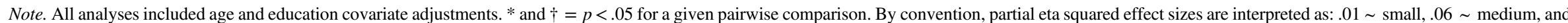
$.14 \sim$ large (Murphy \& Myors, 2004). Bonferroni adjustments were made for each ANCOVA or MANCOVA but no overall experiment-wise adjustments were made. 
differences among groups on most of the neurocognitive tests. Notably, some of the group mean score comparisons were statistically significant despite non-significant omnibus tests (see Table 2). The statistically significant group differences after Bonferroni adjustment are as follows: For the memory subtests, List Learning Short Delay Free Recall mean scores were significantly higher for CIV compared to BNTBI and BMTBI groups; List Learning Long Delay Free Recall mean scores were significantly higher for the CIV group compared to the BMTBI group; and Name, Address, and Phone scores were significantly higher for the NBNTBI group compared to the BMTBI group. For the subtests measuring attention, Digits Forward scores were significantly higher for the CIV group compared to the BMTBI group as were the Number and Letter C Efficiency scores. The Design Construction test measuring spatial abilities was significantly higher for the NBNTBI group compared to the CIV and BMTBI groups. Finally, scores on a Categories test in the domain of executive functioning were significantly higher for the NBNTBI group compared to the CIV group.

\section{Aim 2}

The initial analyses suggested that there were no significant differences on any of the 22 measures of cognitive ability between blast-exposed Veterans who were and were not diagnosed with MTBI (BMTBI vs. BNTBI). Therefore, we assessed the neurocognitive effect of blast exposure alone without regard to MTBI by combining data from the BMTBI and BNTBI groups to form a single blast exposed (BE) group comprising Veterans with $(n=49)$ and without $(n=20)$ MTBI history. We compared the BE group's mean scores with those from the NBNTBI group $(n=23)$. There were statistically significant omnibus test results indicating differences between the BE and NBNTBI groups on measures of Immediate Recall, Delayed Recall, and Design Construction. As shown in Table 3, there were statistically significant differences between BE and NBNTBI groups on List Learning Long Delay Recall, Story Memory Immediate and Delayed Recall, Name Address Phone Delayed Recall, Design Construction, and Categories tests after Bonferroni adjustment. Results for all tests, regardless of statistical significance, indicated that NBNTBI Veterans performed better than the BE Veterans. Sensitivity analyses combining the NBNTBI and CIV groups into a larger non-blast exposed group resulted in no changes in the direction or significance of effects when compared to BE participants.

\section{Aim 3}

To examine whether significant differences in neurocognitive test results were uniquely associated with blast exposure or possibly related to PTSD symptoms, we replicated our Aim 2 analyses in Aim 3, but also included PTSD symptom severity as assessed by the PCL as a covariate. As shown in Table 3, all MANCOVA omnibus tests were non-significant, and the only Bonferroni-adjusted significant differences between the BE and
NBNTBI groups after adjusting for PTSD symptoms were on tests of Story Memory Immediate and Delayed Recall.

\section{DISCUSSION}

Comparison of neuropsychological test performance supported our hypothesis that civilian controls and non-blastexposed OEF/OIF Veterans would perform better than both blast-exposed OEF/OIF Veteran groups. However, the pattern of differences was inconsistent and there were no significant differences between blast-exposed Veterans with MTBI history and blast-exposed Veterans without MTBI history. When both blast-exposed groups were aggregated and compared to non-blast-exposed Veterans with MTBI history, more consistent and robustly significant neuropsychological performance differences were demonstrated between the groups. Consistent with the results of previous studies, subsequent statistical adjustment for PTSD symptom severity eliminated almost all significant neuropsychological performance differences between Veteran groups with the exception of immediate and delayed story recall.

The primary analyses comparing neurocognitive test performance across all four participant groups showed a complex pattern of mixed significant and non-significant results. MANCOVA omnibus tests were significant for measures of attention, spatial abilities, and executive function, but not memory. Some of the mean score comparisons, particularly memory measures, were statistically significant despite nonsignificant omnibus significance tests. The pattern of relative performance across groups was inconsistent across measures and not always supportive of our initial hypotheses. In particular, Design Construction was significantly higher for the NBNTBI group compared to CIV and BMTBI groups, and, similarly, Category Fluency was significantly higher for the NBNTBI group compared to the CIV group. Effect sizes were small to medium and would not be considered clinically significant when examined in aggregate, as none of the average $t$ scores for any groups or subtests were below 44 , although it is possible that performance of individuals might reach clinical significance in some cases. The most consistent pattern was the absence of significant differences between blast-exposed Veterans with MTBI history (BMTBI group) and blastexposed Veterans without MTBI history (BNTBI group).

More robust and consistent performance differences were evident when both MTBI and non-MTBI blast-exposed groups were aggregated and compared to non-blast-exposed Veterans without MTBI history. These analyses revealed significant differences showing impairment associated with blast exposure for multiple measures of learning and memory, as well as design construction and categories. Furthermore, results for all measures, regardless of statistical significance, indicated that Veterans without blast exposure performed better than those who were blast exposed. These results suggest that blast exposure is associated with some cognitive risk, but they do not support that the source of this risk is MTBI history in this sample of Veterans. Results indicated significantly worse performance on story memory 
Table 3. Neuropsychological Assessment Battery (NAB) mean raw score differences for veterans with and without blast exposure

\begin{tabular}{|c|c|c|c|c|c|c|c|c|c|c|}
\hline & \multicolumn{3}{|c|}{ Adjusted for age \& education } & \multicolumn{3}{|c|}{ Adjusted for age, education, \& PCL score } & \multicolumn{2}{|c|}{ Unadjusted raw score mean $(S D)$} & \multicolumn{2}{|c|}{ T-Score mean $(S D)$} \\
\hline & $\mathrm{F}$ & $p$-Value & Partial $\eta^{2}$ & $\mathrm{~F}$ & $p$-Value & Partial $\eta^{2}$ & No blast exposure & Blast exposed & No blast exposure & Blast exposed \\
\hline & \multicolumn{10}{|c|}{ Learning \& Memory } \\
\hline Immediate Recall & 1.72 & 0.15 & 0.05 & 1.35 & 0.26 & 0.06 & & & & \\
\hline List Learning & 2.87 & 0.09 & 0.02 & 1.71 & 0.20 & 0.02 & $25.78(3.38)$ & $23.88(4.26)$ & $51.73(9.75)$ & $47.39(9.31)$ \\
\hline Story Memory & 4.78 & $0.03 *$ & 0.04 & 4.61 & $0.04 *$ & 0.05 & $63.61(12.11)$ & $57.88(11.33)$ & $50.30(10.14)$ & $45.99(9.89)$ \\
\hline Daily Living Memory & 2.89 & 0.09 & 0.02 & 2.37 & 0.13 & 0.03 & $46.65(4.68)$ & $44.04(4.83)$ & $53.17(11.64)$ & $50.93(10.22)$ \\
\hline Medication Instructions & 0.94 & 0.33 & 0.01 & 1.04 & 0.31 & 0.01 & $25.17(2.73)$ & $24.23(2.76)$ & $50.09(10.74)$ & $49.90(9.35)$ \\
\hline Name, Address, Phone & 3.36 & 0.07 & 0.03 & 1.92 & 0.17 & 0.02 & $21.48(2.63)$ & $19.81(3.37)$ & $51.18(9.45)$ & $48.92(10.43)$ \\
\hline Delayed Recall & 2.26 & $0.05 *$ & 0.09 & 1.56 & 0.17 & 0.09 & & & & \\
\hline List Learning, Short Delay & 3.20 & 0.07 & 0.03 & 0.96 & 0.33 & 0.01 & $8.61(1.83)$ & $7.80(2.00)$ & $53.25(10.05)$ & $47.94(10.69)$ \\
\hline List Learning, Long Delay & 4.01 & $0.05^{*}$ & 0.03 & 1.62 & 0.21 & 0.02 & $8.57(2.39)$ & 7.35 (2.48) & $53.06(11.16)$ & $46.32(12.82)$ \\
\hline Story Memory & 5.43 & $0.02 *$ & 0.04 & 4.07 & $0.05 *$ & 0.05 & $34.22(4.78)$ & $30.57(5.84)$ & $50.49(9.44)$ & $45.77(9.05)$ \\
\hline Daily Living Memory & 3.35 & 0.07 & 0.03 & 0.73 & 0.31 & 0.01 & $15.91(2.00)$ & $14.68(2.43)$ & $53.13(7.32)$ & $47.14(12.71)$ \\
\hline Medication Instructions & 0.07 & 0.80 & 0.00 & 0.07 & 0.80 & 0.00 & $8.43(1.12)$ & $8.26(1.13)$ & $50.89(9.22)$ & $49.16(10.66)$ \\
\hline \multirow[t]{2}{*}{ Name, Address, Phone } & 6.58 & $0.01 *$ & 0.05 & 2.16 & 0.15 & 0.03 & $7.48(1.08)$ & $6.42(1.65)$ & $53.22(7.24)$ & $47.00(11.23)$ \\
\hline & \multicolumn{10}{|c|}{ Attention } \\
\hline Digit Span & 1.55 & 0.22 & 0.02 & 0.73 & 0.48 & 0.01 & & & & \\
\hline Digits forward & 2.17 & 0.14 & 0.02 & 1.38 & 0.24 & 0.01 & $9.30(2.40)$ & $8.28(2.39)$ & $52.67(10.08)$ & $45.33(10.66)$ \\
\hline Digits backward & 2.76 & 0.10 & 0.02 & 1.01 & 0.31 & 0.01 & $6.30(2.79)$ & $5.09(2.54)$ & $53.73(11.01)$ & $49.81(10.41)$ \\
\hline Numbers and Letters & 0.60 & 0.55 & 0.01 & 0.15 & 0.86 & 0.00 & & & & \\
\hline C efficiency & 0.83 & 0.36 & 0.01 & 0.00 & 0.99 & 0.00 & $65.14(23.10)$ & $58.23(20.76)$ & $50.87(9.39)$ & $45.65(8.87)$ \\
\hline \multirow[t]{2}{*}{ D efficiency } & 0.64 & 0.43 & 0.01 & 0.22 & 0.64 & 0.00 & $49.91(10.26)$ & $48.42(9.28)$ & $49.86(9.25)$ & $45.74(9.54)$ \\
\hline & \multicolumn{10}{|c|}{ Spatial } \\
\hline \multirow[t]{2}{*}{ Design construction } & 4.22 & $0.04 *$ & 0.03 & 2.05 & 0.16 & 0.02 & $58.35(4.85)$ & $53.88(6.73)$ & $51.38(9.51)$ & $52.86(9.35)$ \\
\hline & \multicolumn{10}{|c|}{ Executive Functioning } \\
\hline Executive Functioning & 1.46 & 0.23 & 0.03 & 0.93 & 0.43 & 0.03 & & & & \\
\hline Mazes & 0.50 & 0.48 & 0.00 & 1.07 & 0.30 & 0.01 & $19.87(4.48)$ & $19.83(5.81)$ & $50.16(8.77)$ & $50.06(8.91)$ \\
\hline Categories & 4.44 & $0.04 *$ & 0.03 & 2.45 & 0.12 & 0.03 & $27.61(7.78)$ & $23.90(7.13)$ & $51.87(8.61)$ & $51.49(6.87)$ \\
\hline Word generation & 0.39 & 0.53 & 0.00 & 0.01 & 0.94 & 0.00 & $11.30(3.02)$ & $10.48(3.77)$ & $50.70(9.25)$ & $47.97(8.21)$ \\
\hline
\end{tabular}

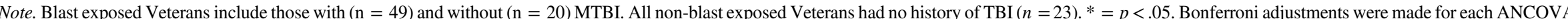
or MANCOVA but no overall experiment-wise adjustments were made. By convention, partial eta squared effect sizes are interpreted as: .01 small, .06 medium, and .14 large (Murphy \& Myors, 2004 ). 
subtests by BE compared to NBNTBI Veteran groups after adjusting for PTSD, which suggests that blast exposure may be associated with reduced ability to recall structured verbal material; however, this finding should be interpreted with caution given the sample selection and small sample size in this study.

Consistent with results of previous studies, when covariate analyses were used to explore the effect of PTSD symptoms on neuropsychological differences between blast exposed and non-blast exposed groups, the majority of previously significant results were no longer significantly different. Indeed, only immediate and delayed story recall score differences remained significant when PCL score was included as a covariate, indicating that PTSD symptoms account for much of the association between blast exposure and cognition. However, further research is needed to evaluate the role of PTSD symptoms in the relationship between blast exposure and neurocognitive test performance because PTSD symptoms were significantly associated with group membership in this study, necessitating a cautious interpretation of these results.

The results of these PCL covariate analyses raise the possibility that the absence of neuropsychological test performance differences between MTBI- and non-MTBI diagnosed blastexposed Veterans is because cognitive effects are mediated by traumatic stress instead of MTBI. These results suggest that blast exposure may increase the risk for traumatic stress, whether or not the blast exposure results in MTBI. Thus, these results are consistent with the hypothesis that the primary source of cognitive risk associated with combat blast exposure is posttraumatic stress, not TBI-induced brain dysfunction. This hypothesis is consistent with the results of several other OEF/OIF Veteran studies that included participants with comorbid MTBI and PTSD (Belanger et al., 2010; Hoge et al., 2008; Verfailie et al., 2014); however, these aggregate findings do not preclude the possibility that some individuals may experience clinically significant cognitive impairment following MTBI.

Another possible explanation for the lack of cognitive differences between MTBI- and non-MTBI diagnosed blast-exposed Veterans may be the definition of MTBI. In particular, concerns have been raised regarding the "disorientation" criterion for combat MTBI diagnosis because disorientation could be a psychological effect of the stress of near-death combat experience rather than a brain dysfunction effect of blast-related neurotrauma (Belanger, Uomoto, \& Vanderploeg, 2009; Iverson, 2010). Indeed, the majority of our MTBI-diagnosed Veterans did not endorse LOC or PTA and were MTBI-diagnosed on the basis of the disorientation. If current diagnostic criteria result in a large number of false positive diagnoses, then it is conceivable that any actual long-term cognitive effects of neurotrauma could be obscured by the statistical noise of the resulting mixed sample. Future studies should examine the potential influence of LOC and PTA, versus disorientation, on cognitive testing in the context of MTBI.

Our results did not support cognitive effects of deployment per se. None of our 22 measures of cognitive ability showed significantly better performance in the civilian group than in the group that was deployed but not blast exposed, a finding contrary to recent research on the effects of deployment (e.g., Vasterling et al., 2006). It is possible, however, that methodological differences or inadequate statistical power due to our small sample size could account for the lack of statistically significant differences among groups. In particular, patterns of results showed slightly worse performance across most cognitive measures by deployed Veterans. Additionally, our control sample, although matched for potentially important characteristics such as age, education, gender, and estimated IQ, was from a civilian sample obtained in different settings and at different times from our Veteran sample. This study did not include a non-deployed Veteran control group which would be needed to further examine the effects of deployment on cognitive test performance. An additional limitation of our study may further limit the generalizability of our findings on deployment effects. Unlike the Vasterling et al. (2006) study, we did not have a longitudinal design that allowed comparison of the effects of deployment within participants. Therefore, the impact of deployment on cognitive functioning warrant replication and further exploration in future studies.

Because of the nature of research with Veterans and members of the military who were injured in combat situations, this research was also limited in some important and practical ways. Our Veteran sample groupings of MTBI diagnoses were based on self-report. Our sample size was limited, particularly with regard to Veterans who were deployed but not blast-exposed. Although consecutively recruited, a large number of our participants were clinically referred and all were a convenience sample recruited in a medical center setting. Additionally, our comparison groups are subject to all the caveats about group comparability and potential for confounded results associated with nonexperimental research. Particularly, the results related to civilian controls should be interpreted with caution as this group is likely very different from our three other Veteran participant groups. Finally, we did not assess cognitive abilities before deployment or blast exposure.

Despite these practical limitations, this study had several strengths. Specifically, we were able to gather extensive information from our participants through comprehensive clinical interviews and test batteries. The assessors who administered neurocognitive tests, although aware that participants were all part of a VA study on blast and MTBI, were blinded to Veteran group status (i.e., they were unaware of whether or not the Veteran they were testing had experienced blast exposure of MTBI history). Our sample is comparable to the general population of OEF/OIF Veterans using VA services in terms of gender and education; although we did not collect data on ethnicity for this study, the general ethnic makeup of the sample reflected Pacific Northwest demographics rather than the national Veteran population. These demographics suggest that our results may be generalizable to a broader population of Veterans with some limitations. We gathered important information about potential contributors to cognitive functioning, such as blast exposure, 
PTSD symptoms, and other clinical characteristics to examine, as comprehensively as possible, the cognitive functioning of individuals with different types of symptom profiles and blast exposure history. Future papers will address additional issues, such as psychodiagnostic group differences, the relationship between cognitive complaints and psychopathology, and the relationship between objective cognitive test performance and subjective cognitive complaints.

Future research should build upon these and other similar findings. First and foremost, research is needed to replicate these findings, including additional and/or different neurocognitive tests, to confirm the pattern of results obtained in this study. Future research should include non-deployed members of the military as a more comparable control group. Future research should further examine the impact of mechanism of injury, and investigators should consider conducting research on blast exposure regardless of MTBI diagnosis due to the potential for misclassification of MTBI status. When examining cognitive outcomes, many researchers adjust for the known confounders of age and education; however, this study and others support the need to include PTSD as a covariate (Verfaellie et al., 2014). Additionally, PTSD treatment studies should systematically examine neuropsychological function as an important and potentially modifiable outcome. Finally, future research should not only present results in aggregate, but present proportions of individuals with clinically significant impairment. We plan to present such findings in future publications in the hopes of providing results in an alternative format more conducive to future resource planning.

This project was undertaken to inform and improve the clinical care that we provide to U.S. Service Members. Clinical implications of this study include the importance of a comprehensive neurocognitive assessment for those exposed to blast, regardless of MTBI diagnosis. Additionally, such comprehensive assessments should include an evaluation of PTSD symptoms, given their potential influence on cognitive functioning.

In summary, the results from this study illustrate a complex picture of cognitive functioning as it relates to blast exposure, MTBI history, and PTSD. Although some of the results suggest that there are deficits associated with combat blast exposure, the information gleaned from the comparisons accounting for PTSD are potentially important for Veterans and U.S. Service Members: Because most of the significant differences in neurocognitive functioning between the groups of blast exposed and non-blast exposed Veterans were eliminated once PTSD was accounted for, results suggest that cognitive impairment may be significantly related to mental health factors. This is hopeful news for our Veterans and U.S. Service Members who are struggling with these diagnoses because there is good evidence to support the effectiveness of treatments for PTSD. Therefore, combining cognitive rehabilitation for MTBI history or blast exposure with effective treatment for PTSD may provide benefit for those struggling with cognitive symptoms following blast exposure or MTBI.

\section{ACKNOWLEDGMENTS}

This work was supported by VA Merit Review Award \#B5060R to D.S. from the Department of Veterans Affairs, Veterans Health Administration, Office of Research and Development, Rehabilitation Research and Development, a Veterans Affairs Health Services Research and Development Center of Innovation grant, Center to Improve Veteran Involvement in Care, Award \#CIN 13-404, and an Agency for Healthcare Research \& Quality-funded PCOR K12 award to MEO (\#1 K12 HS019456 01). This material is the result of work supported with resources and the use of facilities at the Portland Veterans Affairs Medical Center, Portland, Oregon. The contents do not represent the views of the U.S. Department of Veterans Affairs or the United States Government. All authors declare no conflicts of interests involved in the research presented in the manuscript. The corresponding author (M.E.O.) declares she had full access to all the data in the study and takes responsibility for the integrity of the data and the accuracy of the data analysis. D. Storzbach and M. O'Neil contributed equally to this work.

\section{REFERENCES}

American Psychiatric Association. (2000). Diagnostic and statistical manual of mental disorders (Revised 4th ed.). Washington, DC: American Psychiatric Association.

Belanger, H.G., Kretzmer, T., Yoash-Gantz, R., Pickett, T., \& Tupler, L.A. (2009). Cognitive sequelae of blast-related versus other mechanisms of brain trauma. Journal of the International Neuropsychological Society, 15(1), 1-8.

Belanger, H.G., Uomoto, J.M., \& Vanderploeg, R.D. (2009). The Veterans Health Administration system of care for mild traumatic brain injury: Costs, benefits, and controversies. Journal of Head Trauma Rehabilitation, 24(1), 4-13.

Belanger, H.G., Kretzmer, T., Vanderploeg, R.D., \& French, L.M. (2010). Symptom complaints following combat-related traumatic brain injury: Relationship to traumatic brain injury severity and posttraumatic stress disorder. Journal of the International Neuropsychological Society, 16, 194-199.

Brenner, L.A., Terrio, H., Homaifar, B.Y., Gutierrez, P.M., Staves, P.J., Harwood, J.E., ... Warden, D. (2010). Neuropsychological test performance in soldiers with blast-related mild TBI. Neuropsychology, 24(2), 160-167.

Greve, K.W., Ord, J., Curtis, K.L., Bianchini, K.J., \& Brennan, A. (2008). Detecting malingering in traumatic brain injury and chronic pain: A comparison of three forced-choice symptom validity tests. The Clinical Neuropsychologist, 22(5), 896-918.

Hoge, C.W., McGurk, D., Thomas, J.L., Cox, A., Engel, C.C., \& Castro, C.A. (2008). Mild traumatic brain injuries in U.S. soldiers returning from Iraq. The New England Journal of Medicine, 358, 453-463.

Iverson, G.L. (2010). Clinical and methodological challenges with assessing mild traumatic brain injury in the military. The Journal of Head Trauma Rehabilitation, 25(5), 313-319.

Lange, R.T., Pancholi, S., Brickell, T.A., Sakura, S., Bhagwat, A., Merritt, V., \& French, L.M. (2012). Neuropsychological outcome from blast versus non-blast: Mild traumatic brain injury in US military service members. Journal of the International Neuropsychological Society, 18(3), 595.

Lippa, S.M., Pastorek, N.J., Benge, J.F., \& Thornton, G.M. (2010). Postconcussive symptoms after blast and nonblast-related mild traumatic brain injuries in Afghanistan and Iraq war veterans. Journal of the International Neuropsychological Society, 16(5), 856-866. 
Luethcke, C.A., Bryan, C.J., Morrow, C.E., \& Isler, W.C. (2010). Comparison of concussive symptoms, cognitive performance, and psychological symptoms between acute blast-versus nonblast-induced mild traumatic brain injury. Journal of the International Neuropsychological Society, 17(1), 36.

Mac Donald, C.L., Johnson, A.M., Wierzechowski, L., Kassner, E., Stewart, T., Nelson, E.C., ... Brody, D.L. (2014). Prospectively assessed clinical outcomes in concussive blast vs nonblast traumatic brain injury among evacuated US Military personnel. JAMA Neurology, Advance online publication. doi: 10.1001/ jamaneurol.2014.1114

Murphy, K.R., \& Myors, B. (2004). Statistical power analysis (2nd ed.). New Jersey: Erlbaum.

Nelson, W.N., Hoelzle, J.B., Doane, B.M., McGuire, K.A., FerrierAuerbach, A.G., Charlesworth, M.J., ... Sponheim, S.R. (2012). Neuropsychological outcomes of U.S. veterans with report of remote blast-related concussion and current psychopathology. Journal of the International Neuropsychological Society, 18, $1-11$.

Reynolds, C.R., \& Kamphaus, R.W. (2003). Reynolds intellectual screening test. Lutz, FL: Psychological Assessment Resources.

Sheehan, D., Shytle, D., Milo, K., Janavs, J., \& Lecrubier, Y. (2006). MINI International Neuropsychiatric Interview. English Version 5.0.

Stern, R.A., \& White, T. (2003). Neuropsychological Assessment Battery: Administration, scoring, and interpretation manual. Lutz, Florida: PAR.
Tombaugh, T.N. (1996). Test of memory malingering. North Tonawanda, New York: MHS.

Vasterling, J.J., Proctor, S.P., Amoroso, P., Kane, R., Heeren, T., \& White, R.F. (2006). Neuropsychological outcomes of army personnel following deployment to the Iraq war. JAMA, 296, 519-529.

Vasterling, J.J., \& Proctor, S.P. (2011). Understanding the neuropsychological consequences of deployment stress: A public health framework. Journal of the International Neuropsychological Society, 17(1), 1-6.

Verfaellie, M., \& Vasterling, J.J. (2009). Memory in PTSD: A neurocognitive approach. Post-Traumatic Stress Disorder, 1, 1-26.

Verfaellie, M., Lafleche, G., Spiro, A., \& Bousquet, K. (2014). Neuropsychological outcomes in OEF/OIF veterans with selfreport of blast exposure: Associations with mental health, but not MTBI. Neuropsychology, 28(3), 337-346. doi: 10.1037/ neu 0000027

Weathers, F., \& Ford, J. (1996). Psychometric review of PTSD checklist (PCL-C, PCL-S, PCL-M, PCL-PR). In B. Stamm (Ed.), Measurement of stress, trauma and adaptation (pp. 250-251). Lutherville, MD: Sidran Press.

Weathers, F.W., Litz, B.T., Herman, D.S., Huska, J.A., \& Keane, T.M. (1993). The PTSD Checklist (PCL): Reliability, validity, and diagnostic utility. 9th Annual Meeting of the International Society for Traumatic Stress Studies. San Antonio, TX.

Wechsler, D. (2001). Wechsler Test of Adult Reading ${ }^{\mathrm{TM}}$ (WTAR ${ }^{\mathrm{TM}}$ ). San Antonio: The Psychological Corp. 